\title{
A SDR Prototype for Backscatter Sensor Networks
}

\author{
Jinrong $\mathrm{Mo}^{1,2}$, Shengbo $\mathrm{Hu}^{1,2}$, Feng $\mathrm{Lv}^{1,2}$, Yanfeng Shi ${ }^{1,2}$ and Tingting Yan ${ }^{1,2}$ \\ ${ }^{1}$ Institute of Intelligent Information, Guizhou Normal University, 550001 \\ Guiyang, China \\ ${ }^{2}$ Center for RFID and WSN Engineering, Department of Guizhou Education, \\ 550001 Guiyang, China \\ 935472997@qq.com,hsb@nssc.ac.cn,619345028@qq.com,94128088@qq.com, \\ 1592624854@qq.com
}

\begin{abstract}
Low cost and low power is one of the important reasons in the wide application of backscatter sensor networks. Based on LabVIEW and USRP platforms, this paper presents and implements a simple and configurable SDR prototype for backscatter sensor networks. The SDR prototype can apply to the multi-sensor environment with low power. The prototype consists of configurable backscatter transceiver with homodyne receiver and sensor nodes. It can reduce the power consumption of system efficiently through harvesting wireless RF energy and waking sensor nodes. The frequency division multiple access method with configurable sub-carrier is proposed, and the constraint conditions of sensing data pulse period for avoiding collision among sensors are presented and proved in this paper. The performance of the prototype is analyzed, and the closed-form solution of the probability of outage under the log-normal channel is also given and proved. Finally, the experiment and simulation show the SDR prototype is effective and feasible. The EVM value is less than $2.8 \%$, and the probability of outage error between numerical analysis and Monte Carlo simulation is less than $1.86 \%$.
\end{abstract}

Keywords: backscatter, wireless sensor network, SDR, probability of outage

\section{Introduction}

Since the advent of backscatter communication, it has been widely used in the RFID (Radio Frequency Identification) system because of its low cost and low power [1, 2, 15]. Similarly, using the principle of the backscatter communication, the sensor nodes transmitter can be simplified into a transistor connected to the antenna. This will greatly reduce the cost and power consumption of wireless communication system. For example, scholars from the University of Washington researched the WISP (Wireless Identification and Sensing Platform) wireless smart sensor system which can harvest RF energy based on backscatter communication. The system is programmable and extensible and it can support the standard UHF RFID protocols. Now, it has been applied in the fields of wireless temperature measurement, the biological signal acquisition [3, 4, 17], etc. In addition, active automotive safety monitoring system based on backscatter communication and wireless energy harvesting has also aroused widespread concern [5-7], such as the tire pressure monitoring system [8]. Undoubtedly, the study and application of BWSN (backscatter wireless sensor network) will attract more and more attention.

BWSN is mainly used for the environment with slow change. Compared with RFID, the BWSN data rate is slower, generally about $10 \mathrm{bps}$. Therefore, the working distance of BWSN is larger, and continuous working duration is longer. The prototype is very easy to implement, because it has neither the function of receiver nor the anti-collision for backscatter sensor nodes. It's also different from the RFID system that has the negotiation 
and synchronization mechanism. But the problem of co-channel interference must be taken into consideration in multi-sensor application environment [18-19]. In view of this, this paper proposes a configurable SDR (software defined radio) prototype for the BWSN based on the FDMA (frequency division multiple access) $[9,20]$ to improve the flexibility and scalability of the wireless communication, and reduce the cost and power consumption.

\section{The Sensor Signal Model of Backscatter}

The structure of backscatter sensor nodes is very simple. As shown in Figure 1, it can be expressed as an antenna which can switch between the open circuit and the short circuit. When the backscatter sensor receives the carrier signal transmitted by backscatter sensor transceiver, the load state of the backscatter sensor nodes changes with the sensor data. This means the reflection coefficient also changes. So, the reflection signal is modulated by sensor data, and the sensor data returns back to the BWSN transceiver through backscatter link.

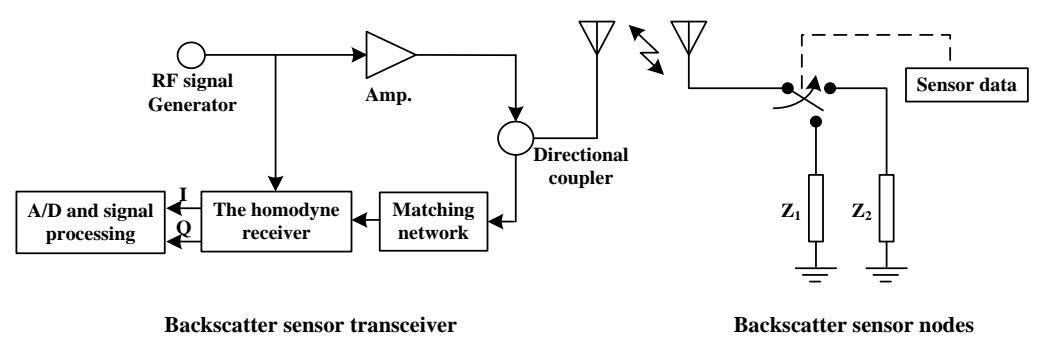

Figure 1. Signal Model of Backscatter Sensor

The carrier complex signal transmitted by a sensor transceiver is given as:

$$
f(t)=\sqrt{2 P} \exp \left[j\left(\omega_{c} t+\varphi_{c}\right)\right]
$$

Where $P, \omega_{c}$ and $\varphi_{c}$ are the peak power, the angular frequency and the phase of the carrier signal respectively.

So, the received complex signal of the $i$-th sensor is

$$
r_{i}(t)=\sqrt{2 P \alpha_{i}} \exp \left[j\left(\omega_{c} t+\varphi_{i}\right)\right]
$$

Where $\alpha_{i}$ is the propagation path loss.

The sensor data changing the antenna load is a binary sequence, so the time-varying reflection coefficient can be expressed as:

$$
\Gamma_{i}(t)=\sum_{k=-\infty}^{\infty} a_{k} p\left(t-k T_{i}\right)
$$

Where $a_{k} \in\{+1,-1\}$ is the binary sequence of the reflection coefficient corresponding to the two load states of the open circuit and the short circuit, and $p(t)$ is the rectangular pulse of sensor data with a cycle of $T_{i}$.

If the sensor reflection efficiency is $\eta$, and the receiving signal power of the $i$-th sensor node by sensor transceiver is $P \eta \alpha^{2}$, the total received complex signals can be written as:

$$
s(t, i)=\sqrt{2 P \eta_{i}} \alpha_{i} \sum_{k=-\infty}^{\infty} a_{k} p\left(t-k T_{i}-\tau_{i}\right) \exp \left[j\left(\omega_{c} t+\varphi_{o i}\right)\right]
$$

Where $\tau_{i}$ is the transmission delay of the $i$-th sensor signal, and $\varphi_{o i}$ is the phase of the $i$-th sensor signal. 
If there are multiple sensors operating simultaneously, the total signals received by transceiver can be represented as:

$$
\begin{aligned}
& s(t)=\sqrt{2 P \eta_{i}} \alpha_{i} \sum_{k=-\infty}^{\infty} a_{k} p\left(t-k T_{i}-\tau_{i}\right) \exp \left[j\left(\omega_{c} t+\varphi_{o i}\right)\right] \\
& +\sum_{j \neq i} \sqrt{2 P \eta_{j}} \alpha_{j} \sum_{l=-\infty}^{\infty} a_{l} p\left(t-k T_{j}-\tau_{j}\right) \exp \left[j\left(\omega_{c} t+\varphi_{o j}\right)\right]+n(t)
\end{aligned}
$$

Where $n(t)$ is the Gaussian white noise.

As stated above, the amplitude and phase of the carrier signal have been modulated by sensor data through reflection coefficient changing. With amplitude and phase modulation of backscattered sensor signals, backscatter modulation communication has been implemented.

Compared with the traditional wireless sensor network, BWSN system has the following characteristics:

1) Because the sensor does not generate local radio-frequency signal and just reflects the carrier signal from the transceiver with no frequency offset, the transceiver is simple to implement and can use homodyne receiver. Also, it is easy to be integrated because of no mirror interference.

2) Since the backscattered sensor signal changes slowly and the low sensor data rate is mostly 10 bps order [21], it can reduce the power consumption of the sensor nodes and increase the transmission distance.

3) Due to the low sensor data rate, the transmission delay can be ignored so that the transceiver and sensors are synchronous. The transceiver and scatter sensors are simple, and the reflected signal only takes amplitude modulation into account. So, we can use ASK modulation to characterize the backscatter communication of sensor nodes.

But the backscatter sensor node doesn't have the mechanism of negotiation and synchronization, the function of receiver and anti-collision. The frequency interference will occur in multi-sensors application environment, which is similar to the collision of multi-tags in RFID system.

\section{The SDR Prototype}

As aforementioned, the structure of backscatter sensor node is simple because of no anti-collision protocol. When multi-sensors work simultaneously, the co-channel interference will occur, which affects wireless access and system performance of the backscatter sensor networks. Consequently, this paper puts forward a SDR prototype for BWSN based on FDMA. The pulse cycle of the sensor data can be configured and the power spectrum aliasing of multiple sensors data is avoided in this prototype. It not only ensures the flexibility of the BWSN but also improves the performance of the system.

\subsection{The Configurable Prototype based on SDR}

Figure 2 shows a generic architecture of SDR, which includes a RF Front end, bandwidth conversion and data processing [9]. The RF Front end consists of antenna, low-noise amplifier, up-converter and down-converter. The up-conversion and down-conversion module is software configurable, e.g., when setting the center frequency. $\mathrm{A} / \mathrm{D}$ and D/A are the two important wideband converters of the SDR architecture. The conversion section determines the bandwidth of the entire system, being the block that influences the method and speed of data processing and the performance of the system. $\mathrm{A} / \mathrm{D}$ and D/A converter should be positioned as close as possible to the antenna in order to optimize performance. Data processing unit is more flexible so that it can be processed by a personal computer (PC), a filed-programmable gate array (FPGA), a digital signal processor (DSP), or the combination of them. Although the structure is flexible in this way, it requires a higher sampling rate, so it is difficult to implement. 


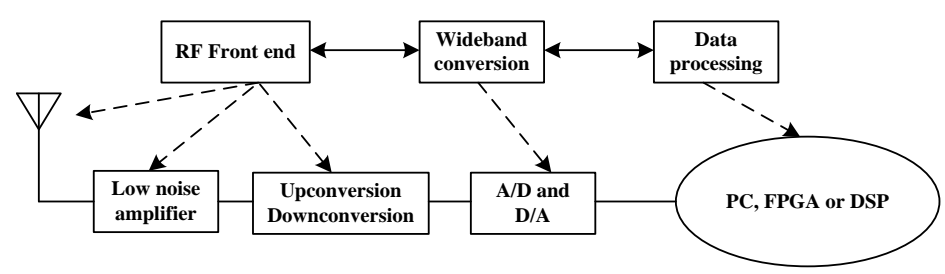

Figure 2. A Architecture of the Generic SDR

A traditional wireless sensor node is shown in Figure 3 (a). The transceiver is a module that realizes the modulation of sensor data, the amplification of RF signals and the transformation of RF signals into digital data. Generally, the transceiver uses hardware to implement the function of physical layer and link layer, and can communicate only with a single protocol and frequency band, but its scalability and flexibility are poor.

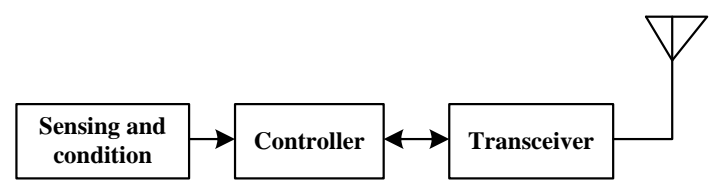

(a) A Traditional Wireless Sensor

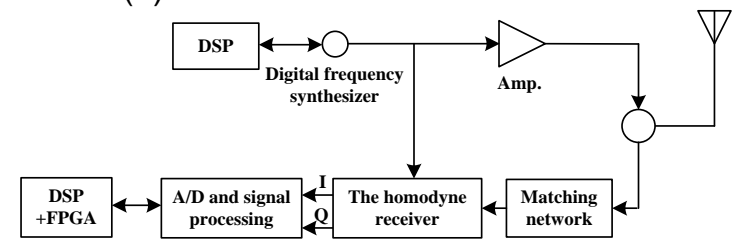

(b) A Configurable Transceiver of Backscatter Sensor

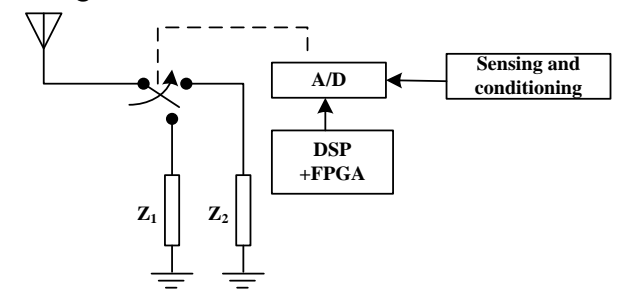

(c) A Backscatter Sensor Node with Configurable Pulse Period

Figure 3. A Configurable Architecture for WSN

In this paper, a configurable architecture for backscatter wireless sensors based on SDR is introduced by two steps. First, the transceiver for backscatter sensors is built by an SDR-based architecture, as illustrated in Figure 3(b). The proposed architecture showed in Figure 3(b) adopts the digital frequency synthesis technology to configure the carrier frequency and meet the requirement of different working frequency bands. The spectrum analysis, filtering and other processing are implemented using FPGA + DSP technology. In addition, the FPGA + DSP technology can support various protocols, and can be interconnected with other network. Second, the backscatter sensors based on software configurable pulse width is constructed. The pulse width of sensor data is configured by using FPGA + DSP technology, as shown in Figure 3(c).

For convenience, the work verifies the feasibility and rationality of the proposed prototype using a mature SDR platform in this paper.

\subsection{The Conditions of Pulse Cycle Configuration}

This paper puts forward an access scheme with sub-carrier modulation, namely the FDMA. It not only avoids the co-channel interference and solves the issue of wireless 
access of multiple sensors, but also makes the structure of backscatter sensor nodes simple.

The power of square wave signal mainly focus on the fundamental wave. For convenience, the received sensor complex signal of ASK modulation can be written by fundamental wave:

$$
s(t, i)=\sqrt{2 P} \alpha_{i} A \cos \left(\omega_{i} t+\varphi_{i}\right) \exp \left[j\left(\omega_{c} t+\varphi_{c i}\right)\right]
$$

Where $\omega_{i}=2 \pi / T_{i}$ is the angular frequency of the sub-carrier for frequency division multiple access.

According to the constraint of the correlated short-range wireless communication spectrum, the power spectrum can be approximated as $[10,16]$ :

$$
S(f) \approx \frac{1}{\left(f-f_{i}\right)^{2}}
$$

Where $f_{i}=1 / T_{i}$.

And the power spectrum of the signal is within $f_{i} \pm r_{i} / 2$, so its bandwidth is given by:

$$
W_{i}=r_{i}=1 / T_{b_{i}}
$$

Where $T_{b}$ is the code-width of sensor data.

Therefore, if the corresponding pulse period of the $1 s t, 2 n d, \ldots$ and $n$-th sensor meets: $T_{1}<T_{2}<\cdots<T_{n}$, and in order to avoid the power spectrum aliasing among sensor data and implement the wireless access of multi-sensors, the corresponding pulse period of the $\mathrm{n}$ sensors should meet the following constraint:

By that analogy:

$$
\frac{1}{T_{b 1}}+\frac{1}{T_{b 2}}<\frac{2}{T_{1}}-\frac{2}{T_{2}}
$$

$$
\frac{1}{T_{b i}}+\frac{1}{T_{b(i+1)}}<\frac{2}{T_{i}}-\frac{2}{T_{(i+1)}}
$$

In the meanwhile, due to the limited spectrum resource, in order to assure the $\mathrm{n}$ sensors data can be received by the transceiver, the corresponding width of $\mathrm{n}$ sensors data element should also meet the following equation:

$$
\sum_{i=1}^{n} \frac{1}{T_{b i}} \leq B
$$

Where $B$ is the bandwidth received by sensing transceiver, and can be chosen as the channel width $250 \mathrm{kHz}$ based on wireless frequency regulations [10].

Equation (10) gives the pulse period constraint to realize non-interfering access of multiple sensors. And Equation (11) gives the upper bound of the accumulated symbol rate of multi-sensors accessed binary data. Using the constraints and the upper bound, the pulse period of multiple sensors data can be configured flexibly to realize noninterference access of multiple backscatter sensors.

\subsection{The RF Front-end of Homodyne Receiver}

As shown in Figure 1, the receiver is co-located with the RF carrier transmitted by transceiver of backscatter wireless sensor. By using the transmitted signal as a reference for homodyne receiving, phase noise cancels out. If we only take a single sensor into account and ignore the thermal noise, the output $I / Q$ signal of the homodyne front-end can be expressed as:

$$
\begin{aligned}
& y_{I}(t)=A(t)_{i} \cos \left(\omega_{i} t+\varphi_{i o}\right) \cos \left(\varphi_{c i}\right) \\
& y_{Q}(t)=A(t)_{i} \cos \left(\omega_{i} t+\varphi_{i o}\right) \sin \left(\varphi_{c i}\right)
\end{aligned}
$$


So the output $I / Q$ signal of the homodyne front-end with multi sensors becomes:

$$
\begin{aligned}
& y_{I}(t)=\sum_{j=1}^{N} A(t)_{j} \cos \left(\omega_{j} t+\varphi_{j o}\right) \cos \left(\varphi_{c j}\right)+n_{I}(t) \\
& y_{Q}(t)=\sum_{j=1}^{N} A(t)_{j} \cos \left(\omega_{j} t+\varphi_{j o}\right) \sin \left(\varphi_{c j}\right)+n_{Q}(t)
\end{aligned}
$$

Where $\mathrm{N}$ is the number of sensors, $n_{I}(t)$ and $n_{Q}(t)$ represent additive independent and identically distributed gaussian noise, the power of noise $\sigma^{2}$ is related to power spectral density $N_{0}$ by:

$$
\sigma^{2}=E\left\{n_{I}^{2}\right\}=E\left\{n_{Q}^{2}\right\}=B N_{0}
$$

Where B is the homodyne RF front-end bandwidth.

\subsection{Data Processing of Homodyne}

After homodyne detecting, the received signal is processed in software. The goal of data processing is to extract the useful sensor data. The process includes signal acquisition and data extraction.

3.4.1. Signal Acquisition: The step of signal acquisition is detailed as follows.

The first step is to sample the homodyne $I / Q$ signal of multi-sensors from Equation (14) and Equation (15) by using Nyquist.

The second step is to calculate the sampled signal by using efficient fast fourier transform (FFT), and estimate the sub-carrier frequency $f_{i}$ for sensor $i$ by using the maximum likelihood estimation method [11].

The last step is to filter the $i$-th sensor data. The frequency response of the filter is described as:

$$
H(f)=\left\{\begin{array}{l}
1, f_{i}-r_{i} / 2<f<f_{i}+r_{i} / 2 \\
0, \quad \text { others }
\end{array}\right.
$$

In order to reduce the complexity of data processing, down-sampling is also performed during the filtering stage.

The signal is extracted after multiplication with $H(f)$. Under the lognormal channel, the signal-to-interference-and-noise ratio is written as:

$$
\operatorname{SINR}=\frac{2 E_{i} h_{i}}{N_{0} / T_{i}+\sum_{j=1, \neq i}^{N} 2 E_{j} h_{j} S\left(f_{j}-f_{i}\right)}
$$

Where $E_{i}$ is the transmitted bit energy. $h_{i}=l_{i} \Omega_{i}, l_{i}$ is the path loss, $\Omega_{i}$ is shadow fading of logarithmic normal distribution, and $\Omega_{i}=e^{X}$. X follows normal distribution $N\left(\mu, \sigma^{2}\right)$.

If the threshold is $\lambda_{t h}$ and $\operatorname{SINR} \geq \lambda_{\text {th }}$, there is no collision among sensors and the non-interfering access of multi-sensor is implemented. On the contrary, there is interference among sensors, namely the collision of sensors occurs, and the non-interfering access of multi-sensor is not realized, that is to say, interruption is inserted.

Generally, the probability of outage is defined as the probability of symbol error rate greater than a certain threshold (typically the order of $10^{-2}$ ) [22]. Under the shadow channel of the lognormal distribution, the probability of outage can be derived (detail in Appendix): 


$$
P_{\text {out }}=1-Q\left(\frac{\ln x-\mu}{\sigma}\right)
$$

3.4.2. Data extraction: In order to extract sensor data, it is necessary to process the acquired $I / Q$ signal. In fact, the corresponding sampling frequency is chosen during down-sampling of the third step of signal acquisition.

According to the constraints for no aliasing of down-sampling spectrum [20, 23]:

$$
\frac{2 f_{U}}{n} \leq f_{s} \leq \frac{2 f_{L}}{n-1}
$$

Where $f_{U}$ and $f_{L}$ are respectively the high and low cut-off frequency of the bandpass signals $y_{I}(t)$ and $y_{Q}(t)$. The bandwidth is $B_{b p}=f_{L}-f_{U}$. And $n$ is a integer.

The sampling rate of Nyquist should meet the constraint of $f_{s} \geq 2 B_{b p}$.

If the down-sampling rate meets:

$$
f_{s}=\frac{\omega_{i}}{2 \pi\left(n+\frac{1}{4}\right)}
$$

The extracted $I$ and $Q$ signals after down-sampling are equivalent to:

$$
\begin{aligned}
& y_{I}\left(k T_{s}\right)=A_{i}\left(k T_{s}\right) \cos \left(\frac{k \pi}{2}+\varphi_{i o}\right) \cos \left(\varphi_{c i}\right) \\
& y_{Q}\left(k T_{s}\right)=A_{i}\left(k T_{s}\right) \cos \left(\frac{k \pi}{2}+\varphi_{i o}\right) \sin \left(\varphi_{c i}\right)
\end{aligned}
$$

Where $k$ is a integer.

The signal level received by transceiver is expressed as:

$$
y\left(k T_{s}\right)=\sqrt{y_{I}^{2}\left(k T_{s}\right)+y_{Q}^{2}\left(k T_{s}\right)}=\left|A_{i}\left(k T_{s}\right) \frac{1 \pm \cos \left(2 \varphi_{i o}\right)}{2}\right|
$$

Thus, if $\varepsilon=\left|A_{i}\left(k T_{s}\right) \frac{1-\cos \left(2 \varphi_{i o}\right)}{2}\right| \quad\left(\varphi_{i o} \neq 0\right)$, the result by exploiting the decoding decision criterion of minimum distance detection [24] is:

$$
a_{k}= \begin{cases}1, & y\left(k T_{s}\right) \geq \varepsilon \\ 0, & \text { others }\end{cases}
$$

Equation (21) to Equation (24) indicate that demodulation and extraction of the sensor data are realized by using the constraint of Equation (20).

\section{Implementation of the SDR Prototype}

In recent years, with the development of software and hardware technology, various kinds of SDR platforms suitable for scientific research are born at the right moment, such as those prototypes of SDR platforms which are implemented by adopting Linrad, High Performance Software Define Radio, GNU Radio and LabVIEW. Among them, LabVIEW and GNU radio are the most commonly used to implement SDR prototype.

\subsection{LabVIEW and GNU Radio}

The GNU Radio is open-source development software to create SDR. It can be operated under the environment of Linux and Windows, and adopts the graphical and scripting programming mode. The applications of GNU Radio are mainly written in Python, while the core signal processing modules are constructed on the microprocessor with $\mathrm{C}++$ floating point arithmetic. Therefore, for GNU radio, the user-specified signal 
processing modules can be developed under $\mathrm{C}++$ environment and SDR platform prototype can be realized in RF front-end with low cost..

LabVIEW is a generic programming system and provides graphical programming environment. It includes a series of function libraries, such as data acquisition, RF communication, signal analysis, digital filtering, data analysis, data display and data storage, etc.. It also supports Windows and provides the graphical user interface and methods for easy graphical and scripting. LabVIEW has the function of Mathscript, which can be written in and call Matlab. For LabVIEW, the user-specified signal processing blocks can be also developed under the $\mathrm{C}++$ environment, and its function is more powerful. Only by relying on the RF front-end can the SDR prototype be implemented.

At present, the USRP is the most commonly used for RF front-end. The NI USRP2930 can receive GPS clock signal. When it transmits and receives signals, the transmitting, receiving, and local oscillator units share the same clock. This improves frequency accuracy and stability of the transmitted carrier [12]. It also reduces the phase jitter of transmission, which is a great importance to the BWSN [13]. Hence, this paper uses the combination of the LabVIEW and NI USRP 2930 to construct the SDR prototype for BWSN in consideration of the compatibility between hardware and software.

\subsection{NI USRP2930}

The NI USRP2930 completes the function of physical layer, and connects to a host PC installed LabVIEW to act as a SDR prototype. NI USRP2930 has two SMA connectors. One is running in a full duplex mode, the other is only working on one-way patterns of receiving. For the receiving mode, the RF signals enter into USRP2930 by the standard SMA interface, and are mixed down using a direct-conversion receiver to baseband $I / Q$ components, which are sampled by a 2-channel,100MS/s,14-bit analog-to-digital converter (ADC). The digitized $I / Q$ data follows parallel paths through a digital down-conversion (DDC) process that filters and decimates the input $100 \mathrm{MS} / \mathrm{s}$ signal to a user-specified rate. Finally, the user-specified $I / Q$ signals are passed to the host computer over a standard Gigabit Ethernet connection. For the transmission mode, baseband $I / Q$ signals are synthesized by the host computer and fed to the USRP2930 over Gigabit Ethernet. The USRP2930 hardware interpolates the incoming signal to $100 \mathrm{Mbps}$ using a digital up-conversion (DUC) process and then converts the signal to analog with 16-bit, $400 \mathrm{MS} / \mathrm{s}$ sample rate digital-to-analog converter (DAC). Last, the resulting analog signals are mixed up to the specified carrier frequency transmitting via the antenna. As shown in Figure 4. 


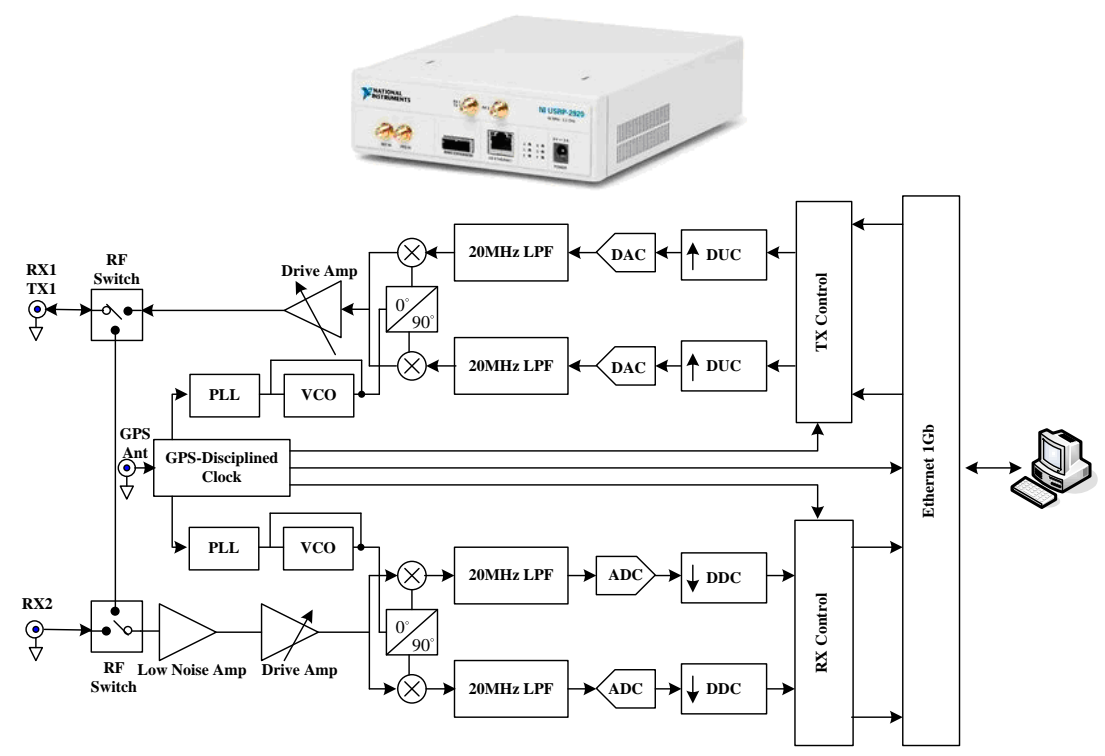

Figure 4. A Block of NI USRP 2930

NI USRP2930 works in the range $50 \mathrm{MHz}$ to $2.2 \mathrm{GHz}$ with tunable center frequency and up to a $25 \mathrm{MHz}$ instantaneous bandwidth, which covers the frequency range including broadcast FM, GPS, GSM, radar and ISM. It also integrates a GPS clock unit for generating a $10 \mathrm{MHz}$ reference clock with high frequency accuracy and stability. The transmitting, receiving, and local oscillator units share the $10 \mathrm{MHz}$ reference clock.

\subsection{A Configurable and RF Waking up Prototype of Backscatter Sensor Network}

A configurable prototype of backscatter sensor network based on USRP is shown in Figure 5. The prototype consist of two NI USRP2930, one data acquisition card of NI USB6216, one module of WISP wireless energy harvesting and two PCs.

As shown in Figure 5, a PC loading LabVIEW and NI USRP2930 implements a prototype of backscatter sensor transceiver based on SDR. This structure transmits the continuous carrier, receives the sensor signals and extracts the sensor data, etc.. Meanwhile, it can generate and transmit RF signals, receive the backscattered signal and generate the baseband I/Q signals. Another PC combines with NI USRP2930, NI USB6216 data acquisition card, WISP RF energy harvesting module [5], and the sensor with its signal conditioning circuit. This is a prototype of the backscatter sensor node for implementing the configuration of the pulse period (sub-carrier frequency). The backscatter sensor node prototype also acquires and transmits the sensor data. WISP is a wireless sensor tag of UHF RF energy harvesting, battery-free and based on backscatter communication. RF energy supplies DC voltage with $1.8 \mathrm{~V}$ to sensors through voltage-multiplying circuit and rectifier circuit of WISP. WISP RF energy harvesting module integrating with sensors constitutes backscatter sensor nodes with RF waking. And the PC, NI USRP2930, NI USB6216 and LabVIEW realize the configuration of sensor data pulse period.
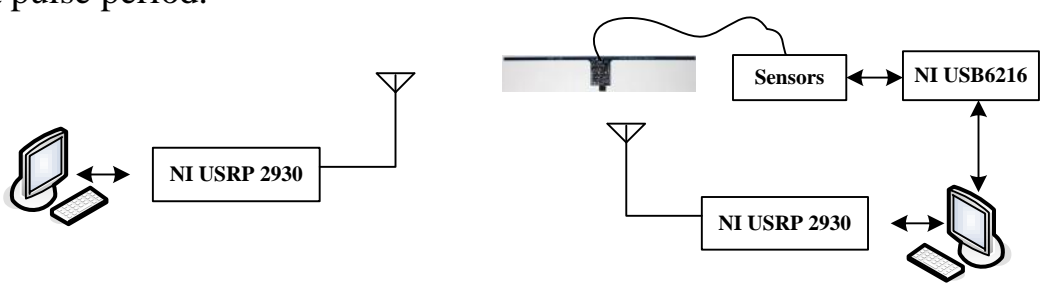

Figure 5. Prototypes of the Configurable Backscatter Sensor Network 


\section{Experiment Setup and Result}

According to the proposed configurable prototype of backscatter sensor in section 4, this section verifies the reasonability and feasibility of the prototype and evaluates the system performance through simulation and experimentation.

\subsection{Experimental Environment}

Experimental environment is shown in Figure 6, which includes two PCs, two NI USRP2930, a oscilloscope of TEK TDS3032C, a WISP RF energy harvesting module, an NI USB6216 data acquisition card and a signal conditioning circuit plate integrating temperature and humidity sensors (TMP20 temperature sensor, HDC1000 humidity sensor).

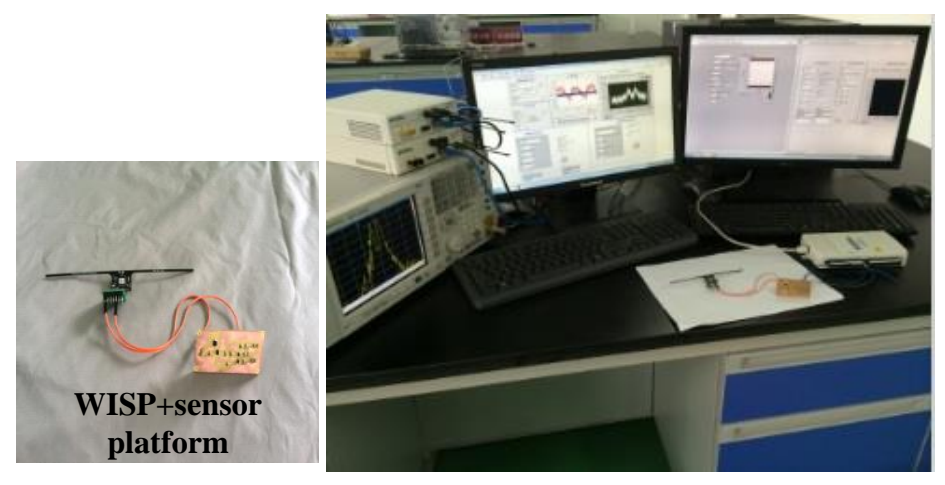

Figure 6. Experimental Environment

Experimental parameters: Carrier frequency is $915 \mathrm{MHz}$, the code-width of sensor data of the temperature and humidity sensors are respectively $0.02 \mathrm{~s}$ and $0.05 \mathrm{~s}$ to satisfy the Equation (11), and the pulse period of sensors data is adjustable.

\subsection{The Results of Simulation and Experimental}

5.2.1. Wireless Energy Harvesting: When the carrier frequency is $915 \mathrm{MHz}$, the transmitting power of USRP is $20 \mathrm{dBmW}$ and the distance between USRP and WISP module is $30 \mathrm{~cm}$, the output voltage waveform of WISP measured by TEK TDS3032C oscilloscope is shown in Figure 7.

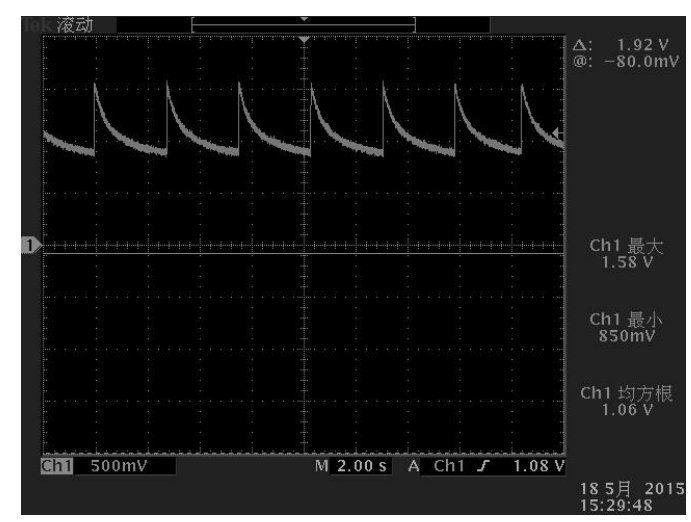

Figure 7. The Output Voltage Wave of WISP 
As shown in Figure 7, the minimum output voltage of WISP is $0.85 \mathrm{~V}$. It can make sensors (TMP20 temperature sensor, HDC1000 humidity sensor) operate so that it realizes the RF waking of sensor nodes.

5.2.2. The received backscatter baseband $I / Q$ signal: The backscatter baseband $I / Q$ signal received by USRP transceiver is shown in Figure 8. In Figure 8(a), only a single node transmits. The received backscatter baseband $I / Q$ signal exhibits two levels, which can be used to distinguish a "1" bit from a "0" bit. In Figure 8(b), two backscatter sensor nodes transmit and the sub-carrier frequency of the sensor data are respectively $20 \mathrm{kHz}$ and $19.98 \mathrm{kHz}$. The backscatter baseband $I / Q$ signal exhibits multiple levels, which indicates that the two nodes collide. In Figure 8(c), two backscatter sensor nodes transmit and the sub-carrier frequency of the sensor data are respectively $20 \mathrm{kHz}$ and $1 \mathrm{kHz}$, which satisfies the equation (9), without spectrum aliasing. The received backscatter baseband $I / Q$ signal exhibits two levels which show that the nodes do not collide and have nil interference with each other. The power spectrum of received signal corresponding to above three cases is shown in Figure 9. Only one signal power spectrum is shown in Figure 9(a). There are two signal power spectrums in Figure 9(b), but the aliasing of the spectrums occurs obviously. There are two signal power spectrums in Figure 9(c), the aliasing does not occur.

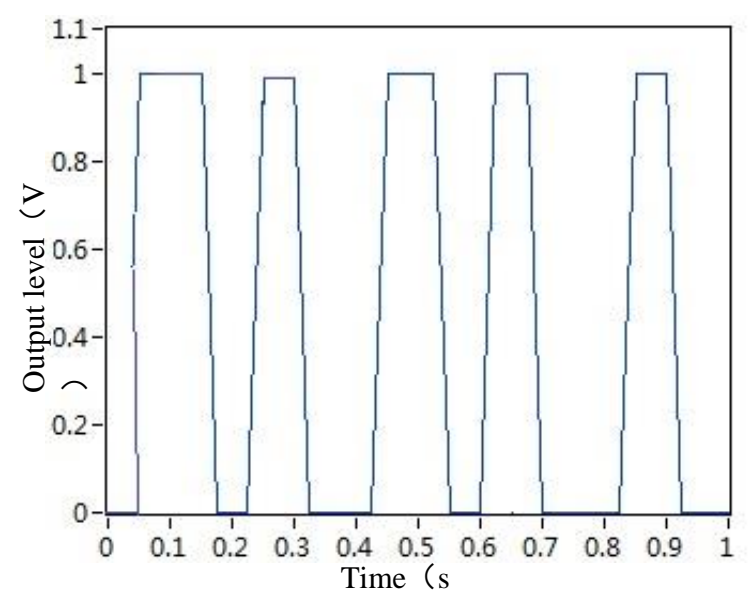

(a) A single node transmission

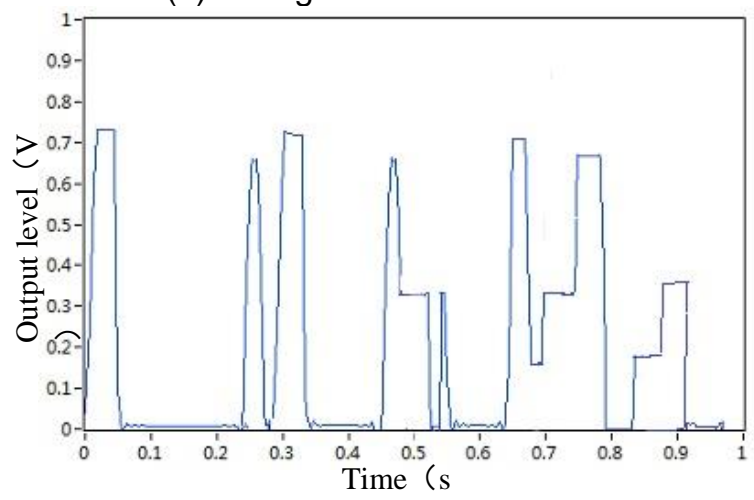

(b) Collision of two nodes transmission 


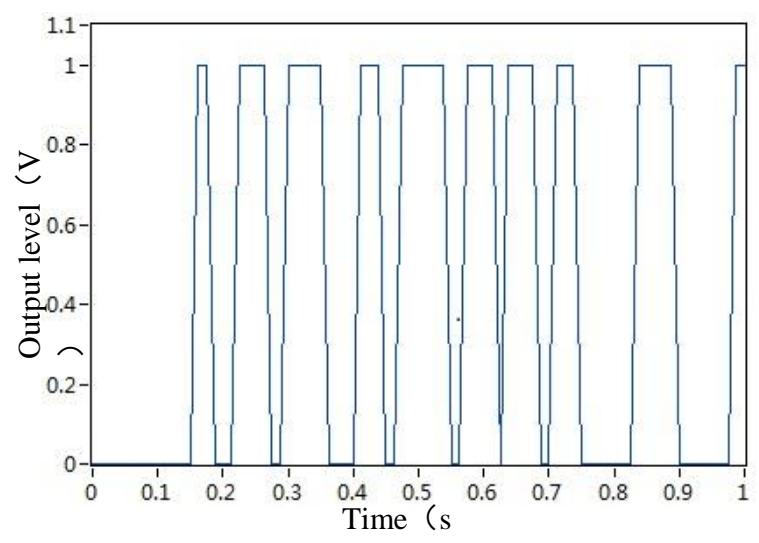

(c) No collision of two nodes transmission

Figure 8. The Received Baseband Signal of Backscatter

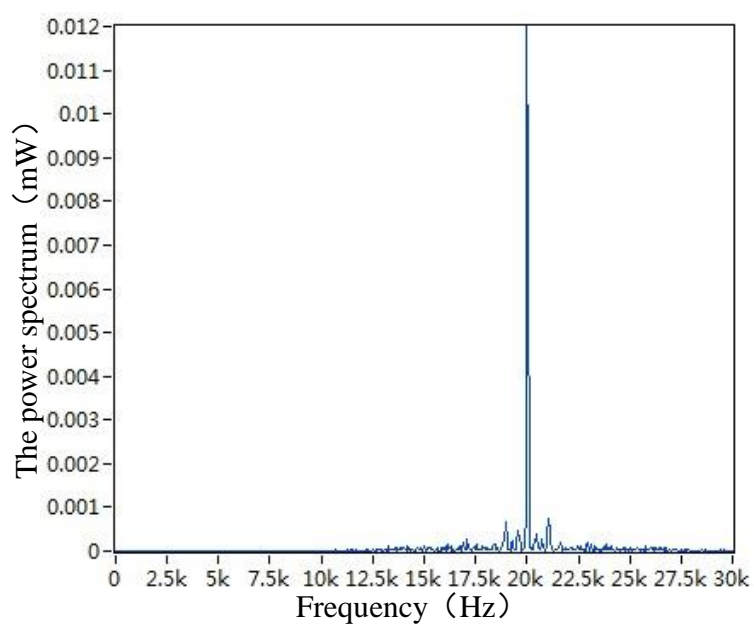

(a) The power spectrum of a single signal

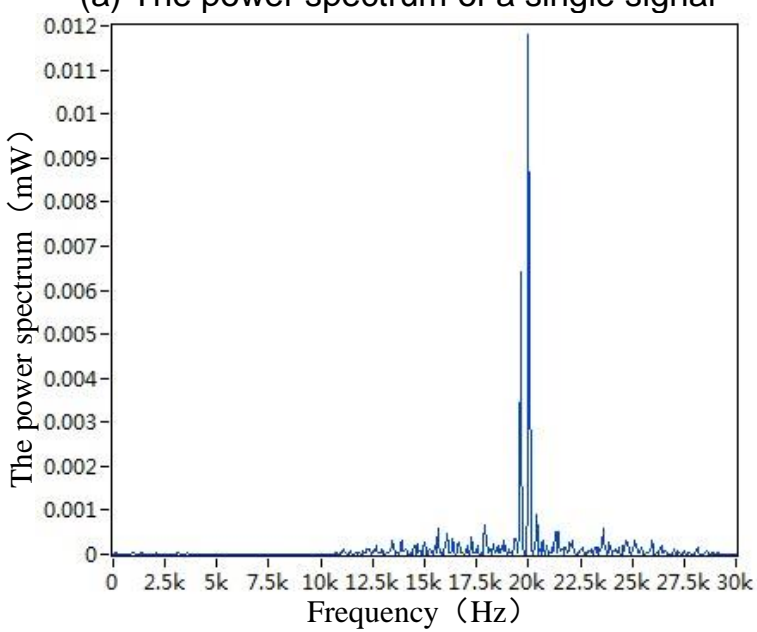

(b) The power spectrum of two signals with aliasing 


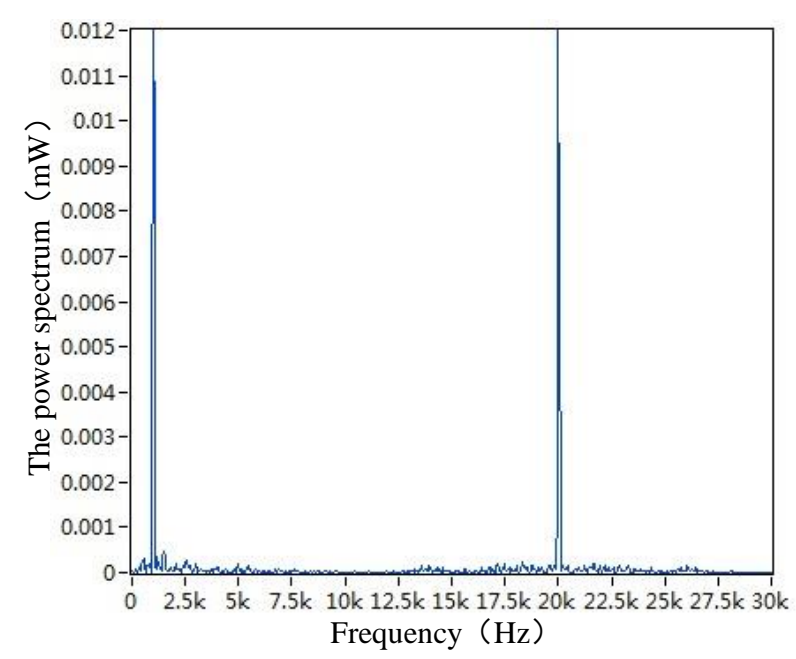

(c) The power spectrum of two signals without aliasing

\section{Figure 9. The Power Spectrum of the Received Signal}

5.2.3. Error Vector Magnitude: Error vector magnitude (EVM) is a description of the totality index of the RF system frequency, phase and amplitude. According to ITU standards [14] and the proposed architecture in this paper, EVM can be defined as:

$$
E V M=\sqrt{\frac{\frac{1}{N} \sum_{i=1}^{N}\left(I_{i}-\hat{I}_{i}\right)^{2}+\left(Q_{i}-\hat{Q}_{i}\right)^{2}}{\frac{1}{N} \sum_{i=1}^{N}\left(\hat{I}_{i}^{2}+\hat{Q}_{i}^{2}\right)}}
$$

Where $\hat{I}_{i}$ and $\hat{Q}_{i}$ are respectively the output baseband $I / Q$ signal of USRP sensor nodes, while $I_{i}$ and $Q_{i}$ are respectively the output baseband $I / Q$ signal of USRP transceiver.

If the carrier frequency ranges from $910 \mathrm{MHz}$ to $915 \mathrm{MHz}$, and the transmission power of USRP sensor node is $20 \mathrm{dBmW}$, the measured value of EVM is $2.8 \%$.

5.2.4. The probability of outage: According to the definition of the probability of outage, $\lambda_{t h}$ is the SINR(signal-to-interference -plus-noise ratio) corresponding to threshold of the symbol error rate. And the SNR (Signal Noise Ratio) of ASK modulation under the Gaussian channel is corresponding to some error bit rate as a reference of $\lambda_{\text {th }}$. So, when the error bit rate of ASK modulation under the Gaussian channel is $1 \times 10^{-2}$, the $\lambda_{\text {th }}$ can be selected $10.34 \mathrm{~dB}$. Other parameters refer to 3GPP specification [25], namely $E_{i} T_{i} / N_{0}=44.7 d B, l_{i}=-1 d B, \mu=1, \sigma=0.5$.

Two users are taking into consideration, when $f_{i}=1 \mathrm{k}$, and $f_{j}$ is at the range of $100 \mathrm{~Hz}$ $\sim 2 \mathrm{kHz}$, the result of Monte Carlo simulation and numerical calculation of the probability of outage for 70,000 times is shown in Figure 10. In Figure 10, the error between the numerical calculation and Monte Carlo simulation is less than $1.86 \%$. 


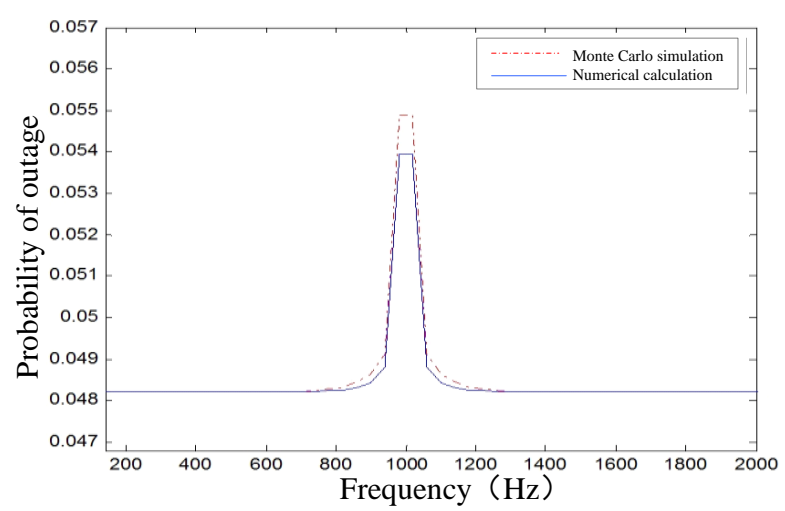

Figure 10. The Monte Carlo Simulation and Numerical Calculation of Outage Probability

\section{Conclusion}

Based on SDR, this paper presents a configurable and simple architecture for BWSN. Besides it is a multi-sensor-oriented application with low power, and it has the function of RF waking. The prototype can be applied to different situations because of its strong flexibility, low cost and low power.

In order to verify the effectiveness and feasibility of the proposed architecture, this paper builds an architecture prototype based on LabVIEW and USRP platform and gives the constraints of collision-free access of multiple sensors. The experimental result shows that the collision-free and nil interfering access of multiple sensors can be realized by configuring the sub-carrier frequency flexibly. Finally, the results of experiment and simulation indicate that the EVM value is less than $2.8 \%$, and the probability of outage error between numerical analysis and Monte Carlo simulation is less than $1.86 \%$, which lays the foundation for the next phase work.

\section{Appendix: The probability of outage derivation}

Under the background of this research, the multiple sensors are co-location, and share a transmitter and transceiver, so it can be assumed $h_{i}=h_{j}$ and $E_{i}=E_{j}$. Consequently, equation (18) can be rewritten as:

$$
\operatorname{SINR}=\frac{1}{\frac{N_{0}}{2 E_{i} T_{i}} l_{i}^{-1} \Omega_{i}^{-1}+\sum_{j=1, \neq i}^{N} S\left(f_{j}-f_{i}\right)}
$$

For the given threshold of signal-to-interference-plus-noise ratio $\lambda_{t h}$, the probability of outage can be expressed as:

$$
\begin{gathered}
P_{\text {out }}=P\left\{\operatorname{SINR}<\lambda_{\text {th }}\right\}=P\left\{\Omega_{i}<\frac{N_{0}}{2 E_{i} T_{i} l_{i}} \frac{\lambda_{\text {th }}}{1-\lambda_{\text {th }} \sum_{j=1, \neq i}^{N} S\left(f_{j}-f_{i}\right)}\right\} \\
\text { If } x=\frac{N_{0}}{2 E_{i} T_{i} l_{i}} \frac{\lambda_{\text {th }}}{1-\lambda_{\text {th }} \sum_{j=1, \neq i}^{N} S\left(f_{j}-f_{i}\right)}, \\
P_{\text {out }}=1-Q\left(\frac{\ln x-\mu}{\sigma}\right)
\end{gathered}
$$

Where $Q(t)=\frac{1}{\sqrt{2 \pi}} \int_{t}^{\infty} e^{-u^{2} / 2} d u$. 


\section{Acknowledgments}

The authors would like to express sincere gratitude to the editor and reviewers for their valuable comments, corrections, and suggestions, which led to an improved version of the original paper. This research is a project partially supported by the National Natural Science Foundation of China (Grant no. 61362004), Guizhou Science and Technology Innovation Group for RFID \& WSN.

\section{References}

[1] H. Stockman, "Communication by means of reflected power", Proceedings of IRE, vol. 36, no. 10, (1948), pp.1196-1204

[2] L. Li and C. Liu, "An Improved Algorithm of LEACH Routing Protocol in Wireless Sensor Networks", Journal of Harbin University Science and Technology, vol. 2, no. 20, (2015), pp. 75-79.

[3] A. Sample, D. Yeager and P. Powledge, "Design of an RFID-based battery-free programmable sensing platform", IEEE Transactions on Instrumentation and Measurement, vol. 57, no. 11, (2008), pp. 2608-2615.

[4] D. Yeager, F. Zhang and A. Zarrasvand, "A $9 \mu \mathrm{A}$, addressable Gen2 sensor tag for biosignal acquisition", IEEE Journal of Solid-State Circuits, vol. 45, no. 10, (2010), pp. 2198-2209.

[5] S. Ergen, A.S. Vincentelli and X. Sun, "The tire as an intelligent sensor", IEEE Transaction on computer-aided design of integrated circuits and systems, vol. 28, no. 7, (2009), pp. 941-955.

[6] J. Grosinger, "Feasibility of backscatter RFID systems on the human body", EURASIP Journal on Embedded Systems, no. 2, (2013), pp. 1-10.

[7] H. Shengbo, S. Bing and S. Heng, "Power transmission performance for ultra high frequency embedded RFID system in tire", Transactions of the Chinese society of agricultural engineering, vol. 29, no. 21, (2013), pp. 150-158.

[8] P. V. Bharat, N. Sihna and K. E. Pujitha, "Tire Pressure Monitoring System Using Ambient Backscatter Technology Containing RF Harvesting Circuitry", International Journal of Advance Engineering and Research Development, vol. 6, no. 1, (2014), pp. 1-11.

[9] P. Ferrari, A. Flammini and E. Sisinni, "New Architecture for a Wireless Smart Sensor Based on a Software Defined Radio", IEEE Transaction on Instrumentation and Measurement, vol. 60, no. 6, (2011), pp. 2133-2141.

[10] L. Burgess, "FCC and ETSI requirements for short-range UHF ASK modulated transmitters", High Frequency Electronics, vol. 12, (2005), pp. 28-35.

[11] M. Gul, S. Leena and X. Ma, "PARAFAC-based frequency synchronization for SC-FDMA uplink transmissions", EURASIP Journal on Advances in Signal Processing, (2014), pp. 1-14.

[12] J. Rong and S, Hu, "The designof DQPSK transceiver based on USRP/LabVIEW", Journal of Guizhou Normal University, vol. 32, no. 6, (2014), pp. 96-99.

[13] D. Arnitz, U. Muehlmann and K. Witrisa, "Wideband characterization of backscatter channels: Derivations and theoretical background", IEEE Transactions on Antennas and Propagation, vol. 60, no. 1, (2012), pp. 257-266.

[14] A. Mahmoud and H. Arslan, "Error vector magnitude to SNR conversion for nondata-aided receivers", IEEE Transaction on wireless communications, vol. 8, no. 5, (2009), 2694-2704.

[15] D. Dobkin, "The RF in RFID: Passive UHF RFID in practice", Newnes (Elsevier), (2008).

[16] L. Stuber, "Principles of Mobile Communication", Springer, (2011).

[17] V. T. Talla, M. Buettner and D. Wetherall, "Hybrid analog-digital backscatter platform with high data rate, battery-free sensing", IEEE Topical Meeting on Wireless Sensors and Sensor Networks (WiSNET), (2013).

[18] O. Incel, S. Sulman and P. Jansen, "Multi-Channel Interference Measurements for Wireless Sensor Networks", Proceedings of IEEE Local Computer Network, (2006).

[19] E. Toscano and L. Bello, "Cross-channel interference in ieee 802.15.4 networks", Proceedings of IEEE International Workshop on Factory Communication Systems, (2008).

[20] D. Zhao and W. Serdijn, "Reconfigurable subsampling receiver architecture for wireless body area networks", Proceedings of International Symposium on Personal, Indoor and Mobile Radio Communications, (2011).

[21] J. Wang, H. Hassanieh and D. Katabi. "Efficient and Reliable Low-Power Backscatter Network", Proceedings of ACM SIGCOMM, (2012).

[22] J. Stephen and K. James, "Analytical calculation of outage probability for a general cellular mobile radio system”, Proceedings of IEEE VTC, (1999).

[23] R. Kodali, S. Kondapalli and L. Boppana, "DDC and DUC Filters in SDR platforms", Proceedings of Conference on Advances in Communication and Control Systems, 2013 
[24] J. Lee, J. Toumpakaris and W. Yu, "Optimal detector for discrete transmit signals in Gaussian interference channels", Proceedings of ICC, (2010).

[25] R. Gonsalves, "Evaluation of outage probability in two-tier open access femtocell networks", Proceedings of CSCITA, (2014).

\section{Authors}

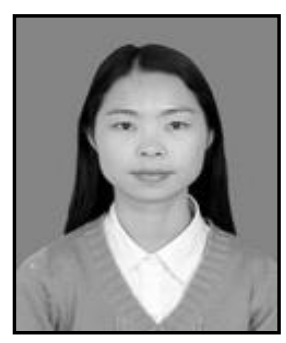

Jinrong Mo, she received her B.Sc. degree in 2013 from Guizhou Normal University, now is studying for her M.Sc. degree in Guizhou Normal University. Her main research interest is sensor network.

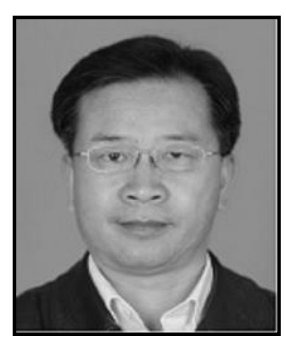

Shengbo Hu, he received his Radio Technology B.Sc. degree in 1985 from Southeast University, received his M.Sc. degree in 1992 from Electronic Engineering Institute of PLA, received his Ph.D. degree in 2006 from Chongqing University, now he is a professor in Guizhou Normal University. His main research interests include intelligent sensing and wireless communication technologies. 